\title{
FACTORS INFLUENCING RECURRENCE IN PATIENTS UNDERGOING LAPAROSCOPIC TREATMENT FOR APPARENT EARLY STAGE CERVICAL CANCER
}

\author{
Artuso V, Pinelli C, Gisone B, Borghi C, Dri M, Casarin J, Ghezzi F \\ University of Insubria, Women's and Children Hospital, Obstetrics and Gynecology, Varese - Italy
}

Objectives. To evaluate oncological outcomes and predictors of recurrence in patients undergoing laparoscopic treatment for apparent early stage cervical cancer (CC).

Methods. A single-centre retrospective study was conducted among patients who had radical surgery for FIGO stage (2009) IA (positive LVSI) - IB1 at Women's and Children Hospital of Varese (Italy) between January 2006 and December 2018. Radical hysterectomy (Querleu and Morrow B-C1 Classification) with or without lymph node dissection according with tumor characteristics. Surgical and oncological outcomes were analyzed.

Results. Among 90 patients who met the inclusion criteria, 12 (13.3\%) had recurrent disease ( 6 vault, 6 pelvis, 3 abdominal, 2 distant, 1 other), and $6(6.7 \%)$ died of disease over the follow-up period (median follow-up 38.2 months). Surgical-related outcomes did not influence survival. Stage of disease has been found to be the main predictor of recurrence $(p=0.03)$, while no association between positive lymph node and relapse was detected. Patients who had preoperative biopsy had a significant higher rate of recurrence in comparison to those undergoing conization $(10 / 45,22.2 \%$ vs $2 / 45$, $4.4 \% ; p=<0.001)$.
After stratification by tumor size, patients with stage IB1 CC undergoing preoperative conization had 0.37 relative risk of recurrence compared to those undergoing cervical biopsy $(10 / 43,23.2 \%$ vs $2 / 23,8.7 \%$; $p=0.14$ ).

Conclusions. Type of preoperative diagnosis might impact on survival in patients undergoing laparoscopic treatment for early stage CC. Further studies are warranted to confirm our findings.

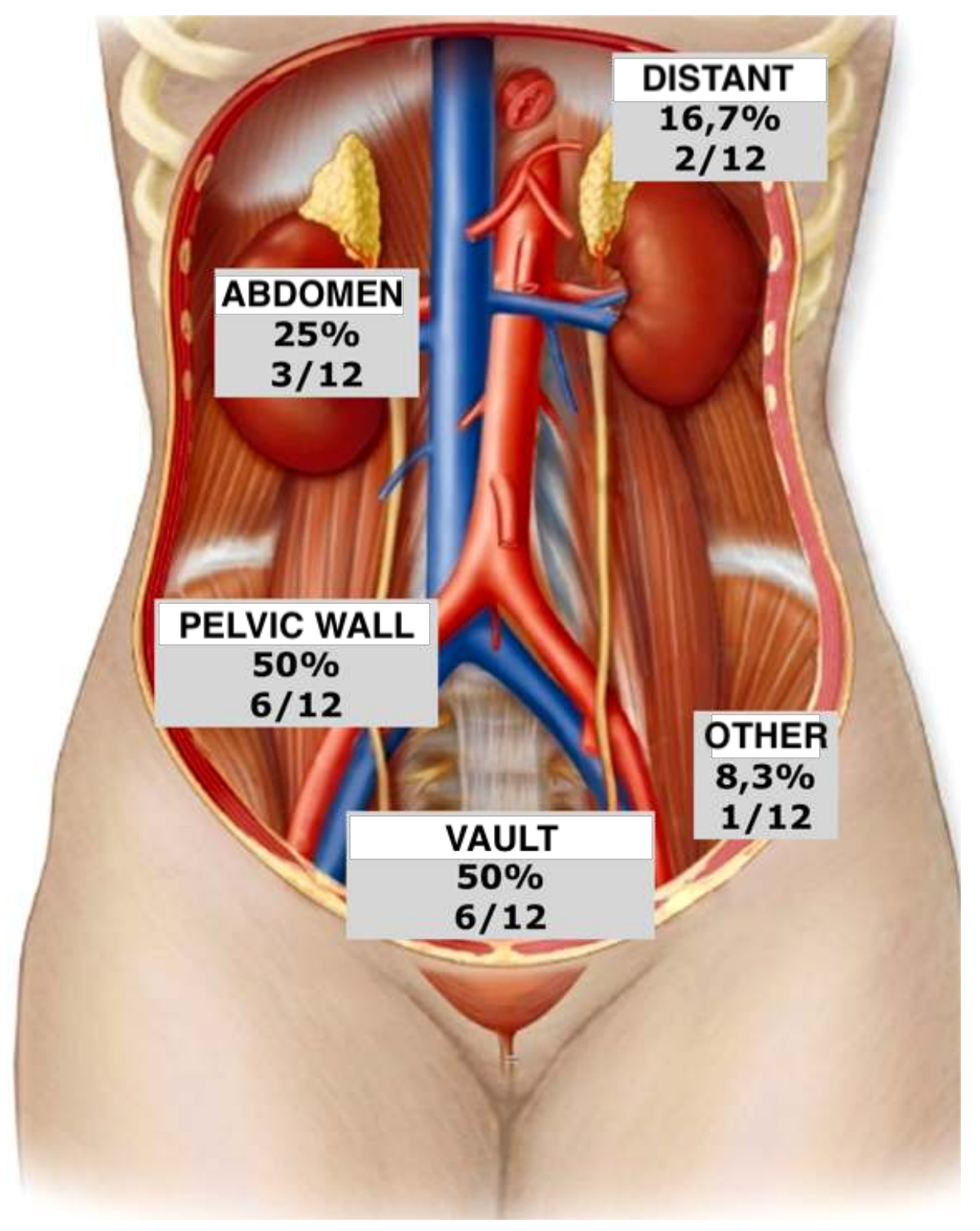

\begin{tabular}{|c|c|c|c|c|c|c|}
\hline & \multicolumn{3}{|c|}{ All population } & \multicolumn{3}{|c|}{ Stage Ib1 (FIGO 2009) } \\
\hline & $\begin{array}{c}\text { Recurrence } \\
\text { YES } \\
N=12\end{array}$ & $\begin{array}{c}\text { Recurrence } \\
N O \\
N=78\end{array}$ & $p$-value & $\begin{array}{c}\text { Recurrence } \\
\text { YES } \\
N=12\end{array}$ & $\begin{array}{c}\text { Recurrence } \\
\mathrm{NO} \\
\mathrm{N}=54\end{array}$ & $p$-value \\
\hline Age (years) & $50(32-67)$ & $42(24-77)$ & .19 & $50(32-67)$ & $42.5(24-77)$ & .28 \\
\hline BMI & $28.0(19.9-39.8)$ & $23.4(16.9-33.4)$ & .08 & $28.0(19.9-39.8)$ & $23.4(17.4-33.4)$ & .07 \\
\hline $\begin{array}{l}\text { Preoperative diagnosis } \\
\text { - Conization } \\
\text { - Cervical Biopsy }\end{array}$ & $\begin{array}{c}2(16.7 \%) \\
10(83.3 \%) \\
\end{array}$ & $\begin{array}{l}43(55.1 \%) \\
35(44.9 \%) \\
\end{array}$ & .01 & $\begin{array}{c}2(16.7 \%) \\
10(83.3 \%)\end{array}$ & $\begin{array}{l}21(38.9 \%) \\
33(61.1 \%)\end{array}$ & .14 \\
\hline Positive node(s) & $1(8.3 \%)$ & $9(11.5 \%)$ & .74 & $1(8,33 \%)$ & $8(14.8 \%)$ & .55 \\
\hline Adjuvant therapy & $8(66.7 \%)$ & $15(19.2 \%)$ & $<0.001$ & $8(66.7 \%)$ & $14(25.9 \%)$ & .007 \\
\hline
\end{tabular}

